\title{
Periodic problem of first order nonlinear uncertain dynamic systems
}

\author{
Yongzhao Wang ${ }^{\mathrm{a}}$, Qian Liu ${ }^{\mathrm{b}}$, Qiansheng Feng ${ }^{\mathrm{c}, *}$ \\ a School of Mathematics and Statistics, Anyang Normal University, Anyang, 455000, China. \\ ${ }^{b}$ School of Civil Engineering and Architecture, Anyang University, Anyang, 455000, China. \\ ${ }^{c}$ Faculty of Mathematics and Physics, Huaiyin Institute of Technology, Huaian, 223003, China. \\ Communicated by Y.-Z. Chen
}

\begin{abstract}
The solution to fuzzy differential equation is very important for solving the uncertainly practical problems in the real world. In this paper, the definition of solution for periodic problems of fuzzy differential equations based on the theory of differential inclusions is given. Using the theory of differential inclusions, function analysis and Kakutani Fixed point theorem, an existence theorem of periodic solutions to first order uncertain dynamical systems is obtained in a more general set. (C)2017 All rights reserved.
\end{abstract}

Keywords: Fuzzy number, uncertain dynamical system, Kakutani fixed point theorem, differential inclusion. 2010 MSC: 47H05, 47H09, 47H10.

\section{Introduction}

Fuzzy differential equations can be studied in three senses:

(1) Hukuhara derivatives ( $\mathrm{H}$-derivatives) and Bede's generalized derivatives which are the generalization of H-derivatives (see [2, 5, 9-12, 14, 16, 18]);

(2) Zadeh's Extension Principle (see [19, 20]);

(3) differential inclusions (see [6-8, 13-15]).

In addition, we know that the theories of fuzzy differential equations are different under these three senses (see $[1,7,15])$. Usually, when one studies practical problems such as uncertain periodic control systems and neural networks with uncertainty, one often needs to consider the following periodic problem of fuzzy differential equation:

$$
\left\{\begin{array}{l}
x^{\prime}=f(t, x) \\
x(0)=x(T)
\end{array}\right.
$$

\footnotetext{
${ }^{*}$ Corresponding author

Email addresses: wangyongzhao1987@126.com (Yongzhao Wang), 357050607@qq.com (Qian Liu), fengqiansheng-2008@163.com (Qiansheng Feng)

doi:10.22436/jnsa.010.12.13
} 
where $f:[0, T] \times \mathbf{E}^{1} \rightarrow \mathbf{E}^{1}$ has at least one fuzzy (non-real) value, i.e., there exists $t_{0} \in[0, T]$ such that $f\left(t_{0}, x\left(t_{0}\right)\right) \in \mathbf{E}^{1} \backslash \mathbf{R}$.

From the results of $[4,7,15]$, we know that there are no periodic solution to fuzzy differential equations in the sense of H-derivatives, so fuzzy differential equations in the senses of H-derivatives cannot be used to describe periodic phenomena in the real world. In this paper, following with [7] and [8], we apply the methods of differential inclusion, functional analysis, set-valued analysis and Kakutani fixed point theorem to consider a periodic problem of fuzzy differential equation in the sense of differential inclusion, i.e., the formula (1.1) holds under the conditions $f:[0, T] \times \mathbf{E}_{c} \rightarrow \mathbf{E}_{c}$ and $\mathbf{E}_{c} \subset \mathbf{E}^{1}$, and the periodic behavior of first order uncertain dynamical system can be seen as:

$$
\left\{\begin{array}{l}
\xi^{\prime} \in f(t, \xi), \\
\xi(0)=\xi(T),
\end{array}\right.
$$

where $f:[0, T] \times \mathbf{R} \rightarrow \mathbf{E}_{\mathcal{c}}$ and for $\eta \in \mathbf{R}, u \in \mathbf{E}_{\mathfrak{c}}, \eta \in u$ means that the membership function of $u$ satisfies $\mu_{u}(\eta)>0$.

The rest of this paper is organized as follows. In Section 2, preliminaries are given. In Section 3, the conditions to existence of solutions to the periodic problem of first order uncertain dynamical systems are figured out, which is simpler and more widely used than previous research work.

\section{Preliminaries}

In this section we recall some facts, which will be used in this paper.

Let $\mathbf{D}^{1}$ be the set of upper semicontinuous normal fuzzy sets with compact supports in $\mathbf{R}$ and $\mathbf{E}^{1}$ be the set of fuzzy convex subsets of $\mathbf{D}^{1}$. For $u \in \mathbf{D}^{1}$ or $\mathbf{E}^{1}$, denote

$$
[u]^{\alpha}=\{\xi \in \mathbf{R} \mid u(\xi) \geqslant \alpha\}, \quad(0<\alpha \leqslant 1), \quad[u]^{0}=\operatorname{cl}\{\xi \in \mathbf{R} \mid u(\xi)>0\},
$$

(see [9]).

Theorem 2.1 ([9]). Let $\left\{A_{\alpha} \subset \mathbf{R} \mid 0 \leqslant \alpha \leqslant 1\right\}$ be a class of nonempty compact sets satisfying

(i) $A_{\beta} \subset A_{\alpha},(0 \leqslant \alpha \leqslant \beta \leqslant 1)$;

(ii) $A_{\alpha}=\bigcap_{n=1}^{\infty} A_{\alpha_{n}}$ for any nondecreasing sequence $\left\{\alpha_{n}\right\}$ in $[0,1]$ satisfying $\alpha_{n} \rightarrow \alpha$.

Then there exists $v \in \mathbf{D}^{1}$ such that $[v]^{\alpha}=A_{\alpha}(0 \leqslant \alpha \leqslant 1)$. Especially $v \in \mathbf{E}^{1}$ if $A_{\alpha}$ is convex. The level set $[v]^{\alpha}$ satisfies (i) and (ii) above, if $v \in \mathbf{D}^{1}$. [v] $]^{\alpha}$ is convex, if $v \in \mathbf{E}^{1}$.

Lemma 2.2. Let $\mathbf{E}_{\boldsymbol{c}}=\left\{\mathfrak{u} \in \mathbf{E}^{1} \mid \mathfrak{u}_{1}(\alpha)=\min [\mathbf{u}]^{\alpha}, \mathfrak{u}_{2}(\alpha)=\max [\mathfrak{u}]^{\alpha}\right.$ are continuous on $\left.[0,1]\right\}$, i.e., $\mathfrak{u} \in \mathbf{E}_{\boldsymbol{c}}$ satisfies the following conditions (i)-(v):

(i) $u$ is normal, i.e., there exists $m \in \mathbf{R}$ such that $u(m)=1$;

(ii) $[u]^{0}$ is bounded in $\mathbf{R}$;

(iii) $u$ is fuzzy convex in $\mathbf{R}$;

(iv) $\mathfrak{u}$ is upper semicontinuous on $\mathbf{R}$;

(v) $u_{1}(\alpha), u_{2}(\alpha)$ are continuous on $[0,1]$.

We call that $u \in \mathbf{E}_{\mathbf{c}}$ is a continuous fuzzy number (fuzzy number in abbreviation).

Lemma 2.3 ([7]). If $\mathrm{u} \in \mathbf{E}_{\mathrm{c}}$, the following (1)-(3) hold: 
(1) $\mathrm{u}_{1}(\alpha), \mathrm{u}_{2}(\alpha)$ are continuous on $[0,1]$;

(2) $\mathfrak{u}_{1}(\alpha)$ is monotone increasing and $\mathrm{u}_{2}(\alpha)$ is monotone decreasing;

(3) $u_{1}(\alpha) \leqslant u_{2}(\alpha)$.

Conversely, if $i(\alpha), s(\alpha):[0,1] \rightarrow \mathbf{R}$, satisfy (1)-(3) above, denote

$$
u(\xi)= \begin{cases}\sup \{\alpha \in[0,1] \mid i(\alpha) \leqslant s(\alpha), & \xi \in[i(0), s(0)]\}, \\ 0, & \xi \notin[i(0), s(0)]\end{cases}
$$

Then there exists $u \in \mathbf{E}_{c}$, such that $[u]^{\alpha}=[i(\alpha), s(\alpha)], u_{1}(\alpha)=i(\alpha), u_{2}(\alpha)=s(\alpha), \alpha \in[0,1]$.

For $u \in \mathbf{E}_{\mathfrak{c}}$, we directly adopt the following parametric representation:

$$
u=\left(u_{1}, u_{2}\right), \quad \text { or } u=\left(u_{1}(\alpha), u_{2}(\alpha)\right), \quad \alpha \in[0,1] .
$$

In this way, a fuzzy number $u \in \mathbf{E}_{\boldsymbol{c}}$ can be directly considered as a point in $\mathrm{C}[0,1] \times \mathrm{C}[0,1]$ or a continuous curve $\left\{\left(u_{1}(\alpha), u_{2}(\alpha)\right) \in \mathbf{R}^{2} \mid \alpha \in[0,1]\right\}$ in $\mathbf{R}^{2}$.

Based on operations $\oplus, \ominus$, and $\otimes$ of Zadeh's extension principle for $u=\left(u_{1}, u_{2}\right), v=\left(v_{1}, v_{2}\right) \in \mathbf{E}_{c}$, $k \in \mathbf{R}$, we have

(1) $u \oplus v=\left(u_{1}+u_{2}, v_{1}+v_{2}\right)$;

(2) $u \ominus v=\left(u_{1}-u_{2}, v_{1}-v_{2}\right)$;

(3) $k \otimes u=\left\{\begin{array}{l}\left(k u_{1}, k u_{2}\right), k \geqslant 0, \\ \left(k u_{2}, k u_{1}\right), k<0 .\end{array}\right.$

Lemma $2.4([7]) . \mathbf{E}_{\mathrm{c}}$ is a closed convex cone in Banach space $X$, and then it is a complete metric space.

Let $\|\cdot\|_{p}$ be Puri's norm on $\mathbf{E}_{\mathfrak{c}}$, then we have $\|u\|=\|u\|_{p}=D(u, 0), u \in \mathbf{E}_{\mathfrak{c}}$, where $D(\cdot, \cdot)$ is the usual Hausdorff metric on $\mathbf{E}^{1}$ (see [23]).

Definition 2.5 ([7]). Let $f:[0, T] \rightarrow \mathbf{E}_{c}, t_{0} \in[0, T]$. If

$$
\forall \varepsilon>0, \quad \exists \delta>0 \text {, such that }\left\|f(t)-f\left(t_{0}\right)\right\|<\varepsilon \text { whenever } t \in[0, T] \text {, and }\left|t-t_{0}\right|<\delta,
$$

then we say that $f$ is continuous at $t_{0}$. If $f$ is continuous at each point of $[0, T]$, we say that $f$ is continuous on $[0, \mathrm{~T}]$.

Lemma 2.6. Let $f:[0, T] \rightarrow E_{c}, f(t)=\left(f_{1}(t, \alpha), f_{2}(t, \alpha)\right)$ where $f_{i}(t, \alpha)=f_{i}(t)(\alpha), i=1,2, \alpha \in[0,1]$, then $f(t)$ is continuous on $[0, T]$ if and only if $\mathrm{f}_{1}(\mathrm{t}, \alpha)$ and $\mathrm{f}_{2}(\mathrm{t}, \alpha)$ are continuous on $[0, \mathrm{~T}] \times[0,1]$.

Definition 2.7 ([7]). Let $f:[0, T] \rightarrow \mathbf{E}_{c}, t_{0} \in[0, T], \eta \in \mathbf{E}_{c}$. If

$$
\forall \varepsilon>0, \quad \exists \delta>0 \text {, s.t. }\left\|\frac{f(t)-f\left(t_{0}\right)}{t-t_{0}}-\eta\right\|<\varepsilon, \quad \text { when } 0<\left|t-t_{0}\right|<\delta \text { and } \forall t \in[0, T],
$$

then we say that $f$ is relatively derivable at $t_{0}$, denoted by $f^{\prime}\left(t_{0}\right)=\eta$ or $\frac{d}{d t} f\left(t_{0}\right)=\eta$. Especially if $f^{\prime}\left(t_{0}\right) \in \mathbf{E}_{\mathbf{c}}$, then $f$ is derivable at $t_{0}$. If $f$ is relatively derivable at each point of $[0, T]$, we say $f$ is that relatively derivable on $[0, \mathrm{~T}]$.

Lemma 2.8. Let $\mathrm{f}:[0, \mathrm{~T}] \rightarrow \mathrm{E}_{\mathrm{c}}$ be relatively derivable on $[0, \mathrm{~T}]$ and $\mathrm{f}(\mathrm{t})=\left(\mathrm{f}_{1}(\mathrm{t}, \alpha), \mathrm{f}_{2}(\mathrm{t}, \alpha)\right)$, then

$$
f^{\prime}(t)=\left(\frac{\partial}{\partial t} f_{1}(t, \alpha), \frac{\partial}{\partial t} f_{2}(t, \alpha)\right), \quad \forall \alpha \in[0,1]
$$


Definition 2.9 ([7]). Let $f:[0, T] \rightarrow \mathbf{E}_{c}$. If for any partition $\Delta$ of $[0, T]: 0=t_{0}<t_{1}<\cdots<t_{n-1}<t_{n}=T$, and for all $\tau_{k} \in\left[t_{k-1}, t_{k}\right](k=1,2, \cdots, n)$, we have $\lim _{\lambda(\Delta) \rightarrow 0} \sum_{k=1}^{n} f\left(\tau_{k}\right) \Delta t_{k}=J$, where

$$
\lambda(\Delta)=\max _{1 \leqslant k \leqslant n} \Delta t_{k}, \Delta t_{k}=t_{k}-t_{k-1}, \quad(k=1,2, \cdots, n),
$$

then we say that $f$ is integrable on $[0, T]$ and denote $J=\int_{0}^{T} f(t) d t$.

Lemma 2.10. Let $f:[0, T] \rightarrow \mathbf{E}_{c}$ be integrable on $[0, T], f(t)=\left(f_{1}, f_{2}\right)$, then

$$
\int_{0}^{T} f(t) d t=\left(\int_{0}^{T} f_{1}(t, \alpha) d t, \int_{0}^{T} f_{2}(t, \alpha) d t\right), \quad \forall \alpha \in[0,1]
$$

and $\int_{0}^{T} f(t) d t \in \mathbf{E}_{c}$.

Lemma 2.11. Let $\mathrm{f}:[0, \mathrm{~T}] \rightarrow \mathbf{E}_{\mathrm{c}}$ be continuous on $[0, \mathrm{~T}]$, then $\mathrm{f}$ is integrable on $[0, \mathrm{~T}]$, and

$$
\left\|\int_{0}^{T} f(t) d t\right\| \leqslant \int_{0}^{T}\|f(t)\| d t .
$$

Lemma 2.12 ([7]). Let $\mathrm{f}:[0, \mathrm{~T}] \rightarrow \mathrm{E}_{\mathrm{c}}$ be continuous on $[0, \mathrm{~T}]$, then

$$
\left(\int_{0}^{t} f(\tau) d \tau\right)^{\prime}=f(t), \quad t \in[0, T]
$$

\section{Periodic problems of first order nonlinear uncertain dynamical systems}

In this section, we study the following periodic problem to first order nonlinear uncertain dynamical system:

$$
\left\{\begin{array}{l}
\xi^{\prime}(t) \in f(t, \xi(t)), \\
\xi(0)=\xi(T),
\end{array}\right.
$$

where $I=[0, T](T>0), f: I \times \mathbf{R} \rightarrow \mathbf{E}_{c}$. Here for $\eta \in \mathbf{R}, \mathfrak{u} \in \mathbf{E}_{\mathfrak{c}}, \eta \in u$ means $u(\eta)=\mu_{\mathfrak{u}}(\eta)>0$, where $\mu_{u}(\eta)$ is the membership function of $u$.

Consider the following periodic problems of differential inclusions:

$$
\left\{\begin{array}{l}
\xi^{\prime}(t) \in[f(t, \xi(t))]^{\alpha}, \\
\xi(0)=\xi(T) .
\end{array}\right.
$$

Definition 3.1. Let $f: I \times \mathbf{R} \rightarrow \mathbf{E}_{c}$. For any fixed $\alpha \in[0,1]$, if $\xi(t)$ is absolutely continuous, $\xi(0)=\xi(T)$ and $\xi^{\prime}(t) \in[f(t, \xi(t))]^{\alpha}$ a.e. on $I$, then we call that $\xi(t)$ is a solution of (3.2) and

$$
\sum_{\alpha}(I ; t)=\{\xi(t) \mid \xi(t) \text { is a solution of }(3.2)\}, \quad(0 \leqslant \alpha \leqslant 1),
$$

is the set of solutions of (3.2). If there exists $v: I \rightarrow \mathbf{D}^{1}$ such that $[v(t)]^{\alpha}=\sum_{\alpha}(I ; t)(t \in I, 0 \leqslant \alpha \leqslant 1)$, then we call that $v(t)(t \in I)$ is a solution of (3.1).

Definition 3.2 ([3]). Let $Y$ and $Z$ be Hausdorff topological spaces. We say that the set-valued mapping $\mathrm{G}: \mathrm{Y} \rightarrow 2^{\mathrm{Z}} \backslash\{\emptyset\}$ is upper semicontinuous, if for any nonempty closed subset $\mathrm{C}$ of $\mathrm{Z}$, the set

$$
\mathrm{G}^{-}(\mathrm{C})=\{\mathrm{y} \in \mathrm{Y} \mid \mathrm{G}(\mathrm{y}) \cap \mathrm{C} \neq \emptyset\}
$$

is a closed subset of $\mathrm{Y}$. 
Let $W^{1,1}(I, \mathbf{R})$ be the Sobolev space with the norm $\|x\|_{W 1,1}=\int_{a}^{b}|x(t)| d t+\int_{a}^{b}\left|x^{\prime}(t)\right| d t$ for $x \in W^{1,1}(I, R)$, then $W^{1,1}(I, \mathbf{R})$ is a Banach space and $W^{1,1}(I, \mathbf{R})$ can be compactly embedded into $L^{1}([a, b], \mathbf{R})$ (see [17]).

Lemma 3.3. Let $W_{\mathrm{p}}^{1,1}(\mathrm{I}, \mathbf{R})=\left\{\xi \in \mathrm{W}^{1,1}(\mathrm{I}, \mathbf{R}) \mid \xi(0)=\xi(\mathrm{T})\right\}$. We define a linear operator $\mathrm{L}: \mathrm{W}_{\mathrm{p}}^{1,1}(\mathrm{I}, \mathbf{R}) \rightarrow$ $\mathrm{L}^{1}(\mathrm{I}, \mathbf{R})$ as $\mathrm{L}(\xi)=\xi^{\prime}-\xi$, then $\mathrm{L}$ is invertible.

Lemma 3.4. $\mathrm{L}^{-1}: \mathrm{L}^{1}(\mathrm{I}, \mathbf{R}) \rightarrow \mathrm{L}^{1}(\mathrm{I}, \mathbf{R})$ is a compact operator.

Lemma 3.5 ([22]). Suppose that $\mathrm{K}$ is the nonempty compact convex subset of $\mathrm{X}$, and $\mathrm{F}: \mathrm{K} \rightarrow \mathrm{P}_{\mathrm{kc}}(\mathrm{K})$ is upper semicontinuous, then $\mathrm{F}$ has a fixed point, i.e., there exists $x^{*} \in \mathrm{K}$ such that $\chi^{*} \in \mathrm{F}\left(\mathrm{x}^{*}\right)$.

Lemma 3.6 ([21]). Let $\left\{f_{n}\right\}_{n=1}^{\infty} \subset L^{p}(\Omega, Y), f \in L^{p}(\Omega, Y), f_{n} \stackrel{w}{\rightarrow} f$ and $f_{n}(x) \in G(x) \mu-a . e$. on $\Omega$, where $\mathrm{G}(\mathrm{x}) \in \mathrm{P}_{w \mathrm{k}}(\mathrm{Y}) \mu$-a.e. on $\Omega$, then $\left.\mathrm{f}(\mathrm{x}) \in \overline{\operatorname{conv}}\left(w-\overline{\lim }\left\{\mathrm{f}_{\mathrm{n}}(\mathrm{x})\right\}_{\mathrm{n}} \geqslant 1\right\}\right) \mu$-a.e. on $\Omega$.

Lemma 3.7 ([22]). Let $\mathrm{AC}([\mathrm{a}, \mathrm{b}], \mathbf{R})$ be the set of absolutely continuous functions on $[\mathrm{a}, \mathrm{b}]$, then $\mathrm{x} \in \mathrm{AC}([\mathrm{a}, \mathrm{b}], \mathbf{R})$ if and only if $x \in W^{1,1}([a, b], \mathbf{R})$.

Lemma 3.8 ([22]). Let $\mathbf{B}$ be a separable normed linear space. Then any bounded set in $\mathrm{B}^{*}$ is weakly* sequentially compact.

Denote

$$
\mathcal{A}=\left\{\xi(\cdot) \in \mathrm{C}(\mathrm{I}, \mathbf{R}) \mid \xi^{\prime}(\cdot) \in \mathrm{L}^{\infty}(\mathrm{I}, \mathbf{R}), \quad \mathrm{I}=[0, \mathrm{~T}]\right\} .
$$

Theorem 3.9. Suppose that $\mathrm{f}: \mathrm{I} \times \mathbf{R} \longrightarrow \mathbf{E}_{\mathrm{c}}$ satisfies

(1) $f(t, \xi)$ is continuous on $T \times \mathbf{R}$;

(2) there exists $M>0$ such that $\|f(t, \xi)\| \leqslant M$;

(3) there exists L > 0 such that

$$
\|f(t, \xi)-f(t, \zeta)\| \leqslant L|\xi-\zeta|,
$$

for $\xi, \zeta \in \mathbf{R}, \mathrm{t} \in \mathrm{T}, 0<\mathrm{L}<1$.

Then the set of solutions $\sum_{\alpha}(\mathrm{I} ; \mathrm{t})$ to (3.2) is nonempty.

Proof. First consider the case $\alpha=0$, i.e., consider the following problem:

$$
\left\{\begin{array}{l}
\xi^{\prime}(t) \in F(t, \xi(t)), \\
\xi(0)=\xi(T),
\end{array}\right.
$$

where $F(t, \xi(t))=[f(t, \xi)]^{0}$.

Define the operator $\mathrm{U}: \mathrm{W}_{\mathrm{p}}^{1,1}(\mathrm{I}, \mathbf{R}) \rightarrow 2^{\mathrm{L}^{1}(\mathrm{I}, \mathbf{R})_{w}}$ as

$$
\mathrm{U}(\xi)=\left\{\zeta \in \mathrm{W}_{\mathrm{p}}^{1,1}(\mathrm{I}, \mathbf{R})_{\mathcal{w}} \mid \zeta(\mathrm{t}) \in \mathrm{F}(\mathrm{t}, \xi(\mathrm{t})) \text { a.e. on } \mathrm{I}\right\},
$$

where $L^{1}(I, \mathbf{R})_{w}$ denotes $L^{1}(I, R)$ endowed with the weak topology.

First, let

$$
\mathrm{V}(\xi)=\mathrm{U}(\xi)-\xi,
$$

we will show that for all $\xi \in W_{p}^{1,1}(I, \mathbf{R}), V(\xi)$ is nonempty compact convex set.

For any $\xi \in W^{1,1}(I, \mathbf{R})$, there exists a sequence of simple functions $\left\{\eta_{n}\right\}$ such that $\eta_{n}(t) \rightarrow \xi(t)$ a.e. on I and $\left\|\eta_{n}\right\| \leqslant \xi(t)$ a.e. on I. By condition (1), $F\left(t, \eta_{n}(t)\right)$ admits a measurable selection $\eta_{n}(t)(n=1,2, \cdots)$. By condition (2), $\eta_{\mathfrak{n}}(t)$ is uniformly integrable for all $n \in \mathbf{N}$, together with Dunford-Pettis theorem, there exists a subsequence of $\left\{\eta_{n}\right\}$, which is weakly convergent in $L^{1}(I, R)$. Without loss of generality, suppose that $\eta_{\mathfrak{n}}(t) \stackrel{W}{\rightarrow} \eta \in \mathrm{L}^{1}(\mathrm{I}, \mathbf{R})$, by Lemma 3.6 and condition (1), we have

$$
\eta(t) \in \overline{\operatorname{conv}} \varlimsup\left\{\eta_{n}(t)\right\} \subset \overline{\operatorname{conv}} \varlimsup F\left(t, \eta_{n}(t)\right) \subset F(t, \xi(t)) .
$$

So $\eta(t) \in U(\xi)$, then we define $\eta(t)-\xi(t)=\varphi(t) \in V(\xi)$, so $V(\xi)$ is nonempty. 
For all $\varphi_{1}(t), \varphi_{2}(t) \in V(\xi)$, then there exist $\eta_{1}(t), \eta_{2}(t) \in U(\xi)$ such that

$$
\varphi_{1}(t)=\eta_{1}(t)-\xi(t), \quad \varphi_{2}(t)=\eta_{2}(t)-\xi(t),
$$

and $\eta_{1}(t) \in F(t, \xi(t)), \eta_{2}(t) \in F(t, \xi(t))$, then $\lambda \eta_{1}(t)+(1-\lambda) \eta_{2}(t) \in F(t, \xi(t))$, therefore

$$
\lambda \varphi_{1}(\mathrm{t})+(1-\lambda) \varphi_{2}(\mathrm{t})=\lambda \eta_{1}(\mathrm{t})+(1-\lambda) \eta_{2}(\mathrm{t})-\xi(\mathrm{t}) \in \mathrm{V}(\xi),
$$

i.e., $\mathrm{V}(\xi)$ is convex.

For all $\varphi(t) \in V(\xi)$, then there exists $\eta \in U(\xi)$ such that $\varphi(t)=\eta(t)-\xi(t)$ and $\eta \in F(t, \xi(t))$,

$$
\|\eta\|=d_{H}(F(t, \xi(t)), 0)=d_{H}\left([f(t, \xi(t))]^{0}, 0\right)=D(f(t, \xi), 0)=\|f(t, \xi)\| \leqslant M .
$$

Since $\xi(t)$ is continuous on $I$, then $\xi(t)$ is bounded on $I$, so there exists $M^{\prime}>0$ such that $\|\xi(t)\| \leqslant M^{\prime}$, then $\|\varphi\|=\|\eta(t)-\xi(t)\| \leqslant M+M^{\prime}$, so $V(\xi)$ is uniformly bounded.

For all $\varphi(t) \in V(\xi)$, there exists $\eta \in U(\xi)$ such that $\varphi(t)=\eta(t)-\xi(t)$ and $\eta \in W^{1,1}(I, R)$, then by Lemma $3.7, \eta$ is absolutely continuous on I, i.e.,

$$
\forall \varepsilon>0, \quad \exists \delta>0, \quad \forall t_{1}, t_{2} \in I, \text { when }\left|t_{1}-t_{2}\right|<\delta, \text { we have }\left\|\eta\left(t_{1}\right)-\eta\left(t_{2}\right)\right\|<\frac{\varepsilon}{2 L},
$$

and $f(t, \xi(t))$ is continuous on $[0, T] \times[-M, M]$, and further uniformly continuous, i.e.,

$$
\left\|f\left(t_{1}, \xi\left(t_{1}\right)\right)-f\left(t_{2}, \xi\left(t_{1}\right)\right)\right\|<\frac{\varepsilon}{2}
$$

from $\xi \in W^{1,1}(I, R)$, so $\left\|\xi\left(t_{1}\right)-\xi\left(t_{2}\right)\right\|<\frac{\varepsilon}{2 L}$, then we have

$$
\begin{aligned}
\left\|\eta\left(t_{1}\right)-\eta\left(t_{2}\right)\right\| & \leqslant d_{H}\left(F\left(t_{1}, \xi\left(t_{1}\right)\right), F\left(t_{2}, \xi\left(t_{2}\right)\right)\right)=d_{H}\left(\left[f\left(t_{1}, \xi\left(t_{1}\right)\right)\right]^{0},\left[f\left(t_{2}, \xi\left(t_{2}\right)\right)\right]^{0}\right) \\
& =D\left(f\left(t_{1}, \xi\left(t_{1}\right)\right), f\left(t_{2}, \xi\left(t_{2}\right)\right)\right)=\left\|f\left(t_{1}, \xi\left(t_{1}\right)\right)-f\left(t_{2}, \xi\left(t_{2}\right)\right)\right\| \\
& \leqslant\left\|f\left(t_{1}, \xi\left(t_{1}\right)\right)-f\left(t_{2}, \xi\left(t_{1}\right)\right)\right\|+\left\|f\left(t_{2}, \xi\left(t_{1}\right)\right)-f\left(t_{2}, \xi\left(t_{2}\right)\right)\right\| \\
& <\frac{\varepsilon}{2}+L\left|\xi\left(t_{1}\right)-\xi\left(t_{2}\right)\right|=\varepsilon,
\end{aligned}
$$

and

$$
\left\|\varphi\left(t_{1}\right)-\varphi\left(t_{2}\right)\right\|=\left\|\eta\left(t_{1}\right)-\xi\left(t_{1}\right)-\eta\left(t_{2}\right)+\xi\left(t_{2}\right)\right\| \leqslant\left\|\eta\left(t_{1}\right)-\eta\left(t_{2}\right)\right\|+\left\|\xi\left(t_{1}\right)-\xi\left(t_{2}\right)\right\|<2 \varepsilon,
$$

so $V(\xi)$ is equicontinous.

For any $\varphi_{n}(t) \in V(\xi)$ and $\varphi_{n}(t) \rightarrow \varphi(t) \in \mathrm{L}^{1}(I, R)$, then there exists $\eta_{n}(t) \in U(\xi)$ such that

$$
\varphi_{n}(t)=\eta_{n}(t)-\xi(t), \quad \eta_{n}(t) \rightarrow \xi(t)+\varphi(t)=\eta(t),
$$

and $\eta_{n}(t) \in F(t, \xi(t))$, then $\eta(t) \in \overline{\operatorname{conv}} \varlimsup \overline{l i m}\left\{\eta_{n}(t)\right\} \subset F(t, \xi(t))$, thus $\eta(t) \in U(\xi)$, so $\varphi(t)=\eta(t)-\xi(t) \in$ $V(\xi)$, this means $V(\xi)$ is closed.

Following the above proved, we know that for all $\xi \in W_{\mathbf{p}}^{1,1}(\mathrm{I}, \mathbf{R}), \mathrm{V}(\xi)$ is nonempty compact convex set.

Second, we show that $\mathrm{L}^{-1} \mathrm{~V}: \mathrm{W}^{1,1}(\mathrm{I}, \mathbf{R}) \rightarrow \mathrm{P}_{\mathrm{kc}}\left(\mathrm{W}^{1,1}(\mathrm{I}, \mathbf{R})\right)$ is uppercontinuous.

Let $K$ be a nonempty weakly closed subset of $L^{1}(I, R)$. By Definition 3.2, we need only to show $\mathrm{U}^{-}(\mathrm{K})=\left\{\xi \in \mathrm{L}^{1}(\mathrm{I}, \mathrm{R}) \mid \mathrm{U}(\xi) \bigcap \mathrm{K} \neq \emptyset\right\}$ is a closed subset of $\mathrm{L}^{1}(\mathrm{I}, \mathrm{R})$.

For all $\left\{\xi_{n}(t)\right\} \subset \mathrm{U}^{-}(\mathrm{K})$, then $\mathrm{U}\left(\xi_{n}\right) \cap \mathrm{K} \neq \emptyset$ and $\xi_{n}(\mathrm{t}) \in \mathrm{L}^{1}(\mathrm{I}, \mathrm{R})$. Suppose $\xi_{n}(\mathrm{t}) \rightarrow \xi(\mathrm{t})$ as $n \rightarrow \infty$ a.e. on I. On the other hand, taking $\eta_{n} \in \mathrm{U}\left(\xi_{n}\right) \cap \mathrm{K}$, by $\mathrm{U}\left(\xi_{\mathrm{n}}\right)=\left\{\varphi(\mathrm{t}) \in \mathrm{L}^{1}(\mathrm{I}, \mathrm{R})_{w} \mid \varphi(\mathrm{t}) \in \mathrm{F}\left(\mathrm{t}, \xi_{n}(\mathrm{t})\right)\right\}$ a.e. on $I$, we have $\eta_{n}(t) \in F\left(t, \xi_{n}(t)\right)$, then there exists a subsequence of $\left\{\eta_{n}\right\}$, which is weakly convergent in $L^{1}(I, R)$. Without loss of generality, suppose $\eta_{n}(t) \stackrel{W}{\rightarrow} \eta(t) \in L^{1}(I, R)$, by Lemma 3.6 and condition (1),

$$
\eta(t) \in \overline{\operatorname{conv}} \varlimsup \varlimsup_{n}(t) \subset \overline{\operatorname{conv}} \varlimsup \overline{\lim } F\left(t, \xi_{n}(t)\right) \subset F(t, \xi(t)) \text { a.e. on } I \text {. }
$$


So $\eta(t) \in U(\xi), K$ is closed set and further $\eta(t) \in U(\xi) \cap K$, then $\xi(t) \in U^{-}(K)$, i.e., $U: L^{1}(I, R) \rightarrow 2^{L(I, R)_{w}}$ is upper semicontinuous, thus $\mathrm{V}(\xi)=\mathrm{U}(\xi)-\xi$ is also upper semicontinuous and maps any bounded set into sequentially compact set.

By Lemma 3.3 and Lemma 3.4, we know that $L^{-1}: L^{1}(I, R) \rightarrow L^{1}(I, R)$ is linear compact operator, and $\mathrm{V}$ is upper semicontinuous, so $\mathrm{L}^{-1} \mathrm{~V}$ is also semicontinuous.

Third, we only need to show that for all $\xi \in W^{1,1}(I, R), V(\xi)$ is the subset of $W^{1,1}(I, R)$.

For all $\varphi(t) \in V(\xi)$, there exists $\eta(t) \in U(\xi)$, such that $\eta(t) \in W^{1,1}(I, R)$ i.e., for all $\varepsilon>0$, there exists $\delta>0$, for any limited disjoint open interval $\left(a_{i}, b_{i}\right)(i=1,2, \cdots, m)$ satisfies $\sum_{i=1}^{m}\left|b_{i}-a_{i}\right|<\delta$, we have $\left|\eta\left(b_{i}\right)-\eta\left(a_{i}\right)\right|<\frac{\varepsilon}{2 m}$, and $\left|\xi\left(b_{i}\right)-\xi\left(a_{i}\right)\right|<\frac{\varepsilon}{2 m}$, then

$$
\begin{aligned}
\sum_{i=1}^{m}\left|\varphi\left(b_{i}\right)-\varphi\left(a_{i}\right)\right| & =\sum_{i=1}^{m}\left|\left(\eta\left(b_{i}\right)-\xi\left(b_{i}\right)\right)-\left(\eta\left(a_{i}\right)-\xi\left(a_{i}\right)\right)\right| \\
& \leqslant \sum_{i=1}^{m}\left|\eta\left(b_{i}\right)-\eta\left(a_{i}\right)\right|+\sum_{i=1}^{m}\left|\xi\left(b_{i}\right)-\xi\left(a_{i}\right)\right|<\varepsilon,
\end{aligned}
$$

thus $\varphi(t)$ is absolutely continuous on $[0, T]$, i.e., $\varphi(t) \in W^{1,1}(I, \mathbf{R})$, so $V(\xi) \subset W^{1,1}(I, \mathbf{R})$, that is $V(\xi)$ is also absolutely continuous.

Thus, since $L^{-1} V: W^{1,1}(I, \mathbf{R}) \rightarrow P_{k c}\left(W^{1,1}(I, R)\right)$ is uppercontinuous, and $V(\xi)$ is the nonempty convex subset of $W^{1,1}(I, \mathbf{R})$, then by Kakutani fixed point theorem (Lemma 3.5), $\mathrm{L}^{-1} \mathrm{~V}$ has a fixed point, that is when $x^{*} \in \mathrm{V}(\xi)$, we have $x^{*} \in \mathrm{L}^{-1} \mathrm{~V}\left(\mathrm{x}^{*}\right)$, i.e., $\mathrm{L}\left(x^{*}\right) \in \mathrm{V}\left(\mathrm{x}^{*}\right)$. Thus $\mathrm{L}\left(\mathrm{x}^{*}\right)=\left(x^{*}(\mathrm{t})\right)^{\prime}-x^{*} \in \mathrm{V}\left(x^{*}\right)=$ $\mathrm{U}\left(\mathrm{x}^{*}\right)-\mathrm{x}^{*}$, so $\left(\mathrm{x}^{*}(\mathrm{t})\right)^{\prime} \in \mathrm{U}\left(\mathrm{x}^{*}\right)$, this means $\left(\mathrm{x}^{*}(\mathrm{t})\right)^{\prime} \in \mathrm{F}\left(\mathrm{t}, \mathrm{x}^{*}(\mathrm{t})\right)$, and $x^{*}(0)=\mathrm{x}^{*}(\mathrm{~T}),\left\{\mathrm{x}^{*}(\mathrm{t})\right\}$ is absolutely continuous, therefore $x^{*}(t)$ is the solution of (3.3), i.e., $\sum_{0}(I ; t) \neq \emptyset$.

In the same way, we can prove that $\sum_{\alpha}(\mathrm{I} ; \mathrm{t}) \neq \emptyset(0<\alpha \leqslant 1)$.

Theorem 3.10. Suppose that $\mathrm{f}: \mathrm{I} \times \mathbf{R} \longrightarrow \mathbf{E}_{\mathrm{c}}$ satisfies

(1) $f(t, \xi)$ is continuous on $I \times \mathbf{R}$;

(2) there exists $M>0$ such that $\|\mathbf{f}(t, \xi)\| \leqslant M$;

(3) there exists L > 0 such that

$$
\|f(t, \xi)-f(t, \zeta)\| \leqslant L|\xi-\zeta|
$$

for $\xi, \zeta \in \mathbf{R}, \mathrm{t} \in \mathrm{T}, 0<\mathrm{L}<1$.

Then the set of solutions $\sum_{\alpha}(\mathrm{I} ; \mathrm{t})$ to (3.2) is uniformly bounded $(0 \leqslant \alpha \leqslant 1)$.

Proof. First consider the case $\alpha=0$, i.e., prove that the set of solutions $\Sigma_{0}(\mathrm{I}, \mathrm{t})$ to (3.3) is uniformly bounded.

By Lemma 3.7, it is obvious that

$$
\Sigma_{0}(\mathrm{I}, \mathrm{t})=\left\{\xi \in \mathrm{W}_{\mathrm{p}}^{1,1}(\mathrm{I}, \mathrm{R}) \mid \xi(\mathrm{t}) \in \mathrm{L}^{-1} \mathrm{~V}(\xi)\right\}
$$

for all $x \in \Sigma_{0}(I, t), x \in L^{-1} V(x)$, that is $L(x)=x^{\prime}-x \in V(x)=U(x)-x$, so $x^{\prime}(t) \in U(x)$, then there exists $y(t) \in U(x)$ such that $x^{\prime}(t)=y(t)$ and $y(t) \in F(t, x(t))$, then $\|y(t)\| \leqslant d_{H}(F(t, x(t)), 0)=\|f(t, x(t))\| \leqslant M$, since $x(t)-x\left(t_{0}\right)=\int_{t_{0}}^{t} x^{\prime}(t) d t=\int_{t_{0}}^{t} y(s) d s$, then $\|x(t)\|=\left\|x\left(t_{0}\right)\right\|+\left|\int_{t_{0}}^{t} y(s) d s\right| \leqslant\left\|x\left(t_{0}\right)\right\|+M T \leqslant M^{\prime}$, i.e., $\Sigma_{0}(\mathrm{I}, \mathrm{t})$ is uniformly bounded.

In the same way, we can show that $\Sigma_{\alpha}(I, t)$ is uniformly bounded $(0<\alpha \leqslant 1)$.

Theorem 3.11. Suppose that $\mathrm{f}: \mathrm{I} \times \mathbf{R} \longrightarrow \mathbf{E}_{\mathrm{c}}$ satisfies

(1) $f(t, \xi)$ is continuous on $I \times \mathbf{R}$;

(2) there exists $M>0$ such that $\|f(t, \xi)\| \leqslant M$; 
(3) there exists L $>0$ such that

$$
\|f(t, \xi)-f(t, \zeta)\| \leqslant L|\xi-\zeta|
$$

for $\xi, \zeta \in \mathbf{R}, \mathrm{t} \in \mathrm{T}, 0<\mathrm{L}<1$.

Then there exists a solution $v: \mathrm{I} \rightarrow \mathbf{D}^{1}$ of (3.1) such that $[v(\mathrm{t})]^{\alpha}=\sum_{\alpha}(\mathrm{I} ; \mathrm{t})(\mathrm{t} \in \mathrm{I}), \alpha \in[0,1]$, and $v(0)=v(\mathrm{~T})$.

Proof. By Theorem 3.9, we know $\sum_{\alpha}(I ; t) \neq \emptyset$. Since $f(t, \xi) \in \mathbf{E}_{\boldsymbol{c}}$, we have $\sum_{\alpha}(I ; t) \subset \sum_{0}(I ; t)(0 \leqslant \alpha \leqslant 1)$.

On the other hand, from Theorem 3.10, we obtain that there exists $M>0$, such that

$$
\forall \xi \in \sum_{\alpha}(\mathrm{I} ; \cdot), \quad\|\xi(\mathrm{t})\| \leqslant M(\mathrm{t} \in \mathrm{I}), \quad \alpha \in[0,1] .
$$

From $\xi^{\prime}(t) \in[f(t, \xi(t))]^{\alpha}$ a.e. on $I$ and $f$ is continuous on $[0, T] \times[-M, M]$, there exists $M^{\prime}>0$ such that $\left|\xi^{\prime}(t)\right| \leqslant M^{\prime}$ a.e. on $\mathrm{I}(0 \leqslant \alpha \leqslant 1)$. For the sake of simplicity, we denote $\sum_{\alpha}=\sum_{\alpha}(\mathrm{I} ; \cdot)(0 \leqslant \alpha \leqslant 1)$.

Obviously, $\left\{\xi(t) \mid \xi \in \sum_{\alpha}\right\}$ is uniformly bounded and equicontinuous on $[0, T]$ and $\left\{\xi^{\prime} \mid \xi \in \sum_{\alpha}\right\}$ is bounded in $\mathrm{L}^{\infty}(\mathrm{I}, \mathbf{R})$.

Since $L^{1}(I, \mathbf{R})$ is a separable Banach space and $\left(L^{1}(I, \mathbf{R})\right)^{*}=L^{\infty}(I, \mathbf{R})$, together with Lemma 3.8, $\left\{\xi^{\prime} \mid \xi \in\right.$ $\left.\sum_{\alpha}\right\} \subset \mathrm{L}^{\infty}(\mathrm{I}, \mathbf{R})$ is weakly* sequentially compact.

For arbitrarily chosen $\left\{\xi_{n}\right\} \subset \sum_{\alpha}$, then there exists a subsequence of $\left\{\xi_{n}\right\}$, which is weakly* convergent. Without loss of generality, we assume $\xi_{n}^{\prime} \stackrel{\mathrm{W}^{*}}{\rightarrow} \zeta \in \mathrm{L}^{\infty}(\mathrm{I}, \mathbf{R})$, i.e., for all $h \in \mathrm{L}^{1}(\mathrm{I}, \mathbf{R})$, we have

$$
\int_{0}^{T} h(t) \xi_{n}^{\prime}(t) d t \rightarrow \int_{0}^{T} h(t) \zeta(t) d t
$$

By Ascoli-Arzelá theorem, there exists a subsequence $\left\{\xi_{n_{k}}\right\}$ of $\left\{\xi_{n}\right\}, \xi \in C(I, R)$ such that

$$
\max _{t \in \mathrm{I}}\left|\xi_{n_{k}}(t)-\xi(t)\right| \rightarrow 0
$$

Taking $h \in \mathrm{L}^{1}(\mathrm{I}, \mathbf{R})$ as

$$
h(s)= \begin{cases}1, & s \in[0, t], \\ 0, & s \notin[0, t], t \in I,\end{cases}
$$

then $\int_{0}^{t} \xi_{n_{k}}^{\prime}(s) d s \rightarrow \int_{0}^{t} \zeta(s) d s(t \in I)$.

As $\xi_{n}(t)$ is absolutely continuous on I $(n=1,2, \cdots)$, we have $\xi_{n_{k}}(t)-\xi_{n_{k}}(0) \rightarrow \int_{0}^{t} \zeta(s) d s$, and further

$$
\xi_{n_{k}}(t) \rightarrow \xi(0)+\int_{0}^{t} \zeta(s) d s, \quad(t \in I) .
$$

Therefore, $\xi(t)=\xi(0)+\int_{0}^{t} \zeta(s) d s(t \in I)$, i.e., $\xi(t)$ is absolutely continuous on $I$ and $\xi^{\prime}=\zeta \in L^{\infty}(I, R)$, and further

$$
\int_{0}^{T} h(t) \xi_{n_{k}}^{\prime}(t) d t \rightarrow \int_{0}^{T} h(t) \xi^{\prime}(t) d t
$$

By $\xi_{n_{k}}^{\prime}(t) \in\left[f\left(t, \xi_{n_{k}}(t)\right)\right]^{\alpha}$ a.e. on I, Lemma 2.10 and Lemma 2.11, for all $[a, b] \subset$ I, we have

$$
\int_{a}^{b} \xi_{n_{k}}^{\prime}(t) d t \in \int_{a}^{b}\left[f\left(t, \xi_{n_{k}}(t)\right)\right]^{\alpha} d t, \quad(n=1,2, \cdots)
$$

After suitably choosing $h \in L^{1}(I, R)$, we can get $\int_{a}^{b} \xi_{n_{k}}^{\prime}(t) d t \rightarrow \int_{a}^{b} \xi^{\prime}(t) d t$, and it is obvious that

$$
\int_{a}^{b} \xi_{n_{k}}(t) d t \rightarrow \int_{a}^{b} \xi(t) d t .
$$


Therefore, by the continuity of $f$ and Lemma 2.8 , we have

$$
\int_{a}^{b} \xi^{\prime}(t) d t \in \int_{a}^{b}[f(t, \xi(t))]^{\alpha} d t .
$$

Then for any $t \in[0, T]$ and $t+\triangle t \in[0, T](\triangle t>0)$ we get

$$
\frac{1}{\Delta t} \int_{t}^{t+\Delta t} \xi^{\prime}(s) d s \in \frac{1}{\Delta t} \int_{t}^{t+\Delta t}[f(s, \xi(s))]^{\alpha} d s .
$$

By the absolute continuity of $\xi(t)$ on $I$, and the continuity of $f$, letting $\Delta t \rightarrow 0^{+}$we obtain $\xi^{\prime}(t) \in$ $[f(t, \xi(t))]^{\alpha}$ a.e. on I. In view of $\xi_{n_{k}}(0)=\xi_{n_{k}}(T)(n=1,2, \cdots)$, it is obvious that $\xi(0)=\xi(T)$ and so $\xi \in \sum_{\alpha}$. Then for any $\left\{\xi_{n}\right\} \subset \sum_{\alpha}$, there exists a subsequence $\left\{\xi_{n_{k}}\right\}$ of $\left\{\xi_{n}\right\}$ and $\xi \in \sum_{\alpha}$ such that

$$
\max _{t \in I}\left|\xi_{n_{k}}(t)-\xi(t)\right| \rightarrow 0, \quad \xi_{n_{k}}^{\prime} \stackrel{w^{*}}{\rightarrow} \xi^{\prime} .
$$

As $L^{1}(I, R)$ is separable, without loss of generality, suppose that there exists $\left\{h_{k}\right\} \subset L^{1}(I, \mathbf{R})$ such that $\overline{\left\{h_{k}\right\}}=L^{1}(I, R)$ and $h_{k} \neq 0(k=1,2, \cdots)$. On $\mathcal{A}$, we introduce the following norm $\|\cdot\|^{*}$ :

$$
\|\xi\|^{*}=\max _{\mathrm{t} \in \mathrm{I}}|\xi(\mathrm{t})|+\sum_{\mathrm{k}=1}^{\infty} \frac{1}{2^{\mathrm{k}}\left\|\mathrm{h}_{\mathrm{k}}\right\|_{1}}\left|\int_{0}^{\mathrm{T}} \mathrm{h}_{\mathrm{k}}(\mathrm{t}) \xi^{\prime}(\mathrm{t}) \mathrm{dt}\right|, \quad \forall \xi \in \mathcal{A} .
$$

Then it is easy to show that $\left(\mathcal{A},\|\cdot\|^{*}\right)$ is a normed linear space and $\sum_{\alpha}$ is bounded subset of $\mathcal{A}$. It is obvious that if $\left\{\xi_{n}\right\} \subset \mathcal{A}, \xi \in \mathcal{A}$ and $\left\{\xi_{n}^{\prime}\right\}$ is bounded in $L^{\infty}(\mathrm{I}, \mathbf{R}),\left\|\xi_{n}-\xi\right\|^{*} \rightarrow 0$ if and only if

$$
\max _{\mathrm{t} \in \mathrm{I}}\left|\xi_{\mathfrak{n}}(\mathrm{t})-\xi(\mathrm{t})\right| \rightarrow 0, \quad \xi_{\mathrm{n}}^{\prime} \stackrel{\mathrm{W}^{*}}{\rightarrow} \xi^{\prime} .
$$

Therefore, from the argument above, $\Sigma_{\alpha}$ is a compact subset of $\mathcal{A}(0 \leqslant \alpha \leqslant 1)$.

For $f: I \times \mathbf{R} \rightarrow \mathbf{E}_{\mathcal{c}}$, it is obvious that $\sum_{\beta} \subset \sum_{\alpha}(0 \leqslant \alpha \leqslant \beta \leqslant 1)$. By the continuity of $f=\left(f_{1}, f_{2}\right)$ on I $\times \mathbf{R}$ and Lemma 2.8, it is immediate to get $\bigcap_{n=1}^{\infty} \sum_{\alpha_{n}}=\sum_{\alpha}$, where $\left\{\alpha_{n}\right\} \subset(0,1]$ is monotone increasing and $\alpha_{n} \rightarrow \alpha \in(0,1]$.

Then by the Stacking Theorem (Lemma 2.2) on Banach spaces, $\sum_{\alpha}$ is the $\alpha$-level set of some fuzzy set $\sum$ on $\mathcal{A}$. In the same way, for each $t \in \mathrm{I}, \Sigma_{\alpha}(\mathrm{I} ; \mathrm{t})$ is the $\alpha$-level set of some fuzzy set on $\mathbf{R}$, i.e., there exists $v: I \rightarrow \mathbf{D}^{1}$ such that $[v(t)]^{\alpha}=\sum_{\alpha}(\mathrm{I} ; \mathrm{t})(\mathrm{t} \in \mathrm{I}), \alpha \in[0,1]$. Therefore there exists a solution $v(\mathrm{t})(\mathrm{t} \in \mathrm{I})$ of (3.1) with $v(0)=v(\mathrm{~T})$.

Example 3.12. In Theorem 3.11, take $I=[0,1], f(t, \xi)=e^{t} u_{0}-\frac{1}{2} \xi, u_{0} \in \mathbf{E}_{\mathcal{c}}$ and $\left[\mathfrak{u}_{0}\right]^{\alpha}=[1+\alpha, 3-\alpha], \alpha \in$ $[0,1]$. Then $\mathrm{f}$ satisfies the conditions in Theorem 3.11. So there exists a periodic solution $v:[0,1] \rightarrow \mathbf{D}^{1}$ to (3.1) with $v(0)=v(1)$. Furthermore, when $f(t, \xi)=e^{t} u_{0}-\frac{1}{2} \xi$, (3.2) becomes

$$
\left\{\begin{array}{l}
\xi^{\prime}(t) \in\left[e^{t} u_{0}-\frac{1}{2} \xi(t)\right]^{\alpha}, \\
\xi(0)=\xi(1)
\end{array}\right.
$$

i.e.,

$$
\left\{\begin{array}{l}
\xi^{\prime}(t) \in e^{t}\left[u_{0}\right]^{\alpha}-\frac{1}{2} \xi(t), \quad \alpha \in[0,1] \\
\xi(0)=\xi(1)
\end{array}\right.
$$

Obviously, the solution to equation

$$
\left\{\begin{array}{l}
\xi^{\prime}(t)=\beta e^{t}-\frac{1}{2} \xi(t), \quad \beta \in\left[u_{0}\right]^{\alpha}, \alpha \in[0,1], \\
\xi(0)=\xi(1),
\end{array}\right.
$$


is $\xi(t)=\frac{2}{3} \beta \cdot e^{-\frac{1}{2} t}\left(\frac{e-1}{1-e^{-\frac{1}{2}}}+e^{\frac{3}{2} t}\right), \beta \in\left[u_{0}\right]^{\alpha}, \alpha \in[0,1]$. Therefore a solution of (3.1) is

$$
v(t)=\frac{2}{3} \cdot e^{-\frac{1}{2} t}\left(\frac{e-1}{1-e^{-\frac{1}{2}}}+e^{\frac{3}{2} t}\right) \otimes u_{0}, \quad(0 \leqslant t \leqslant 1)
$$

with $v(0)=v(1)=\frac{2}{3} \frac{e^{2}-e^{\frac{1}{2}}}{1-e^{\frac{1}{2}}} \otimes \mathrm{u}_{0}$. It is obvious that $v(t)$ is a nonconstant periodic solution to the example.

\section{Acknowledgment}

This work has been supported by the National Natural Science Foundation for Young scientists of China (Grant No. 71502050), the key scientific research projects of colleges and Universities of Henan Province (No. 18A630001 and 18A110010) and the research and cultivation fund of Anyang Normal University (No. AYNU-KP-B10).

\section{References}

[1] R. P. Agarwal, V. Lakshmikantham, J. J. Nieto, On the concept of solution for fractional differential equations with uncertainty, Nonlinear Anal., 72 (2010), 2859-2862. 1

[2] T. Allahviranloo, N. A. Kiani, M. Barkhordari, Toward the existence and uniqueness of solutions of second-order fuzzy differential equations, Inform. Sci., 179 (2009), 1207-1215. 1

[3] J.-P. Aubin, A. Cellina, Differential Inclusions, Spinger-Verlag, Berlin, (1984). 3.2

[4] B. Bede, A note on "two-point boundary value problems associated with non-linear fuzzy differential equations", Fuzzy Sets and Systems, 157 (2006), 986-989. 1

[5] Y. Chalco-Cano, H. Román-Flores, Comparation between some approaches to solve fuzzy differential equations, Fuzzy Sets and Systems, 160 (2009), 1517-1527. 1

[6] Y. Chalco-Cano, H. Román-Flores, Some remarks on fuzzy differential equations via differential inclusions, Fuzzy Sets and Systems, 230 (2013), 3-20. 1

[7] M. Chen, C. Han, Periodic behavior of semi-linear uncertain dynamical systems, Fuzzy Sets and Systems, 230 (2013), 82-91. 1, 1, 2.3, 2.4, 2.5, 2.7, 2.9, 2.12

[8] R. Dai, M. Chen, Some properties of solutions for a kind of semi-linear uncertain dynamical systems, Fuzzy Sets and Systems, 309 (2017), 98-114. 1, 1

[9] P. Diamond, Stability and Periodicity in Fuzzy Differential Equations, IEEE Trans. Fuzzy Syst., 8 (2000), 583-590. 1, 2, 2.1

[10] P. Diamond, Theory and Application of Fuzzy Volterra Integral Equations, IEEE Trans. Fuzzy Syst., 10 (2002), 97-102.

[11] P. Diamond, P. Watson, Regularity of solution sets for differential inclusions quasi-concave in a parameter, Appl Math. Lett., 13 (2000), 31-35.

[12] L. T. Gomes, L. C. Barros, A note on the generalized differences and the generalized differentiability, Fuzzy Sets and Systems, 280 (2015), 142-145. 1

[13] E. Hüllermeier, An approach to modeling and simulation of uncertain dynamical systems, Internat. J. Uncertain. Fuzziness Knowledge-Based Systems, 5 (1997), 117-137. 1

[14] A. Khastan, R. Rodríguez-López, On the solutions to first order linear fuzzy differential equations, Fuzzy Sets and Systems, 295 (2016), 114-135. 1

[15] D. Li, M. Chen, X. Xue, Two-point boundary value problems of uncertain dynamical systems, Fuzzy Sets and Systems, 179 (2011), 50-61. 1,1

[16] V. Lupulescu, Initial value problem for fuzzy differential equations under dissipative conditions, Inform. Sci., 178 (2008), 4523-4533. 1

[17] V. G. Maz'ja, Sobolev Spaces, Springer-Verlag, Berlin, (1985). 3

[18] J. J. Nieto, R. Rodriguez-López, D. Franco, Linear first-order fuzzy differential equations, Internat. J. Uncertain. Fuzziness Knowledge-Based Systems, 14 (2006), 687-709. 1

[19] M. Oberguggenberger, Fuzzy and weak solutions to differential equations, Proceedings of the Tenth International Conference IPMU 2004, Perugi Editrice Universite, (2004). 1

[20] M. Oberguggenberger, S. Pittschmann, Differential equations with fuzzy parameters, Math. Comp. Model. Dyn., 5 (1999), 181-202. 1

[21] N. S. Papageorgiou, Convergence theorem for Banach valued integrable multifunctions, Internat. J. Math. Math. Sci., 10 (1987), 433-442. 3.6

[22] W. Rudin, Functional analysis, McGraw-Hill, New York, (1991). 3.5, 3.7, 3.8

[23] C. Wu, G. Wang, Convergence of sequences of fuzzy numbers and fixed point theorems for increasing fuzzy mapping and application, Fuzzy Sets and Systems, 130 (2002), 383-390. 2 